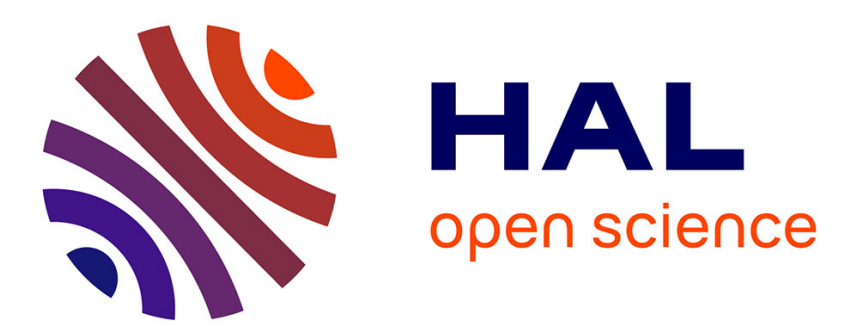

\title{
Equivalent Stress Model for Magnetic Hysteresis Losses Under Biaxial Loading
}

Mahmoud Rekik, Laurent Daniel, Olivier Hubert

\section{To cite this version:}

Mahmoud Rekik, Laurent Daniel, Olivier Hubert. Equivalent Stress Model for Magnetic Hysteresis Losses Under Biaxial Loading. IEEE Transactions on Magnetics, 2014, 50, pp.1 - 4. 10.1109/TMAG.2013.2285241 . hal-01532889

\section{HAL Id: hal-01532889 \\ https://hal.science/hal-01532889}

Submitted on 4 Jun 2017

HAL is a multi-disciplinary open access archive for the deposit and dissemination of scientific research documents, whether they are published or not. The documents may come from teaching and research institutions in France or abroad, or from public or private research centers.
L'archive ouverte pluridisciplinaire $\mathbf{H A L}$, est destinée au dépôt et à la diffusion de documents scientifiques de niveau recherche, publiés ou non, émanant des établissements d'enseignement et de recherche français ou étrangers, des laboratoires publics ou privés. 


\title{
Equivalent stress model for magnetic hysteresis losses under biaxial loading
}

\author{
Mahmoud Rekik*†, Laurent Daniel ${ }^{\dagger \ddagger}$, and Olivier Hubert* \\ *LMT-Cachan (ENS Cachan, CNRS (UMR8535), UPMC, PRES Universud Paris), 94235 Cachan, France \\ ${ }^{\dagger}$ LGEP (SUPELEC, CNRS (UMR8507), UPMC, Univ Paris-Sud), 91192 Gif-sur-Yvette, France \\ ${ }^{\ddagger}$ School of Materials, University of Manchester, M1 7HS, Manchester, UK \\ email: rekik@1mt.ens-cachan.fr, laurent.daniel@u-psud.fr, hubert@1mt.ens-cachan.fr
}

\begin{abstract}
The concept of equivalent stress for hysteretic behaviour is discussed. Magnetic measurements are first performed on iron-silicon thin sheets submitted to biaxial stress. Both anhysteretic and dissipative magnetic responses are recorded. An anhysteretic equivalent stress for the magnetic susceptibility is first applied. A configuration effect allows building an equivalent stress for the coercive field. The combination of the two formulations leads to a prediction of losses in the stress plane.
\end{abstract}

\section{INTRODUCTION}

The present research is motivated by the design of rotors for Variable Frequency Generators (VFG). The increased power density of these devices requires a higher rotation speed (typically 24,000 rpm), leading to higher levels of centrifugal forces and stress in the rotor. A first point is to ensure the mechanical strength of the materials. A second point is to take into account changes in the magnetic behaviour (and ultimately torque) when a high intensity multiaxial stress state is applied. Non-conventional experiments are performed on cross-shaped iron-silicon thin sheets in order to apply biaxial stress representative of the loadings experienced by rotors of VFGs. These experiments are performed on a multiaxial testing machine. Both anhysteretic and dissipative magnetic responses to magneto-mechanical loadings have been recorded (magnetic susceptibility $\chi$, coercive field $H_{c}$, power losses $P$ ). The results allow identifying the most critical stress configurations for this material in terms of iron losses. The results are also used to assess the relevance of an equivalent stress approach. For that purpose a previous definition of equivalent stress $\sigma_{e q}$ for anhysteretic behaviour [1] is recalled and an extension to dissipative behaviour is proposed. Modelling results are finally compared to experiments.

\section{MAgnetic BehaViour UNDER BiAXial STRESS}

\section{A. Experimental set-up}

Measurement of magnetic behaviour is performed on a cross-shaped specimen loaded in tension-compression along two perpendicular directions [1], [2]. A local frame $(1,2)$ is attached to the sheet with direction 1 and 2 corresponding to the rolling direction (RD) and to the transverse direction (TD)

Acknowledgment: the authors wish to thank Arcelormittal for providing the material and 3MT FUI-OSEO Project for financial support. respectively. The local stress tensor $\left(\sigma_{1}, \sigma_{2}\right)$ is calculated from the forces applied along the loading axes $\left(F_{1}, F_{2}\right)$ using a finite element mechanical simulation. Uniformity of both stress and magnetic field has been verified in the area of measurement. Both anhysteretic and hysteretic behaviour of the material are measured. Hysteresis loops have been recorded under a triangular magnetic field waveform $H(t)$ in order to get a constant magnetic field rate. A wide range of first harmonic frequency has been tested up to $800 \mathrm{~Hz}$ with maximal magnetic field $H_{\text {max }} \approx 1000 \mathrm{~A} / \mathrm{m}$. Because the proposed study deals with quasi-static behaviour, only experiments at $f=5 \mathrm{~Hz}$ are reported here. Magnetic field is applied along RD and at $45^{\circ}$ between RD and TD. These two configurations give an insight into some aspects of material anisotropy and allow testing the robustness of the model.

\section{B. Influence of a biaxial stress on the anhysteretic behaviour}

The influence of a biaxial stress on the anhysteretic behaviour is discussed first in terms of secant susceptibility $\chi=(M / H)$. Fig.1 and 2 plot the relative variation of secant susceptibility defined by $(1)$ in the $\left(\sigma_{1}, \sigma_{2}\right)$ plane. The results are reported for the two magnetic loading directions (RD and $\left.45^{\circ}\right)$ and for two magnetic field magnitudes $(H=200 \mathrm{~A} / \mathrm{m}$ and $H=1000 \mathrm{~A} / \mathrm{m})$.

$$
\Delta^{r} \chi=100 \times \frac{\chi\left(\sigma_{1}, \sigma_{2}\right)-\chi(0,0)}{\chi(0,0)}=100 \times \frac{\Delta \chi}{\chi^{0}}
$$

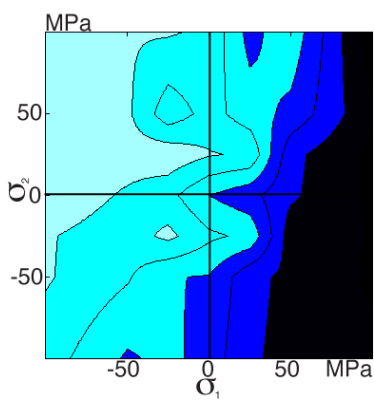

(a) $\vec{H} / / R D$

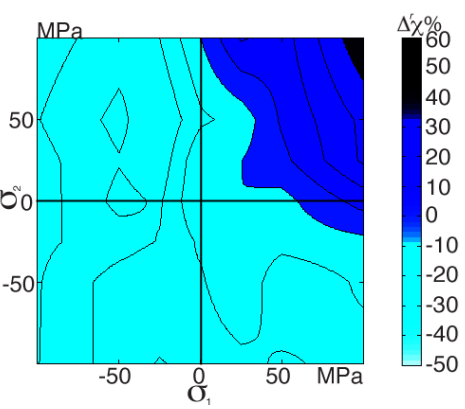

(b) $\vec{H} / / 45^{\circ}$
Fig. 1. Relative variation $\Delta^{r} \chi$ of the secant magnetic susceptibility in the stress plane for a magnetic loading along RD (a) and at $45^{\circ}$ (b) - H=200A/m. 


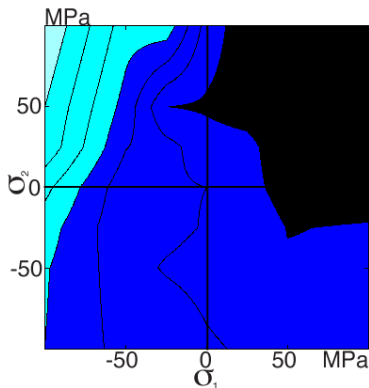

(a) $\vec{H} / / R D$

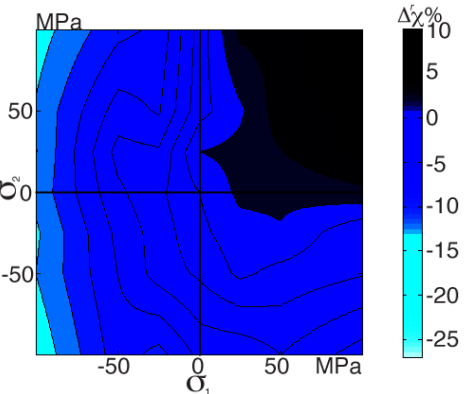

(b) $\vec{H} / / 45^{\circ}$
Fig. 2. Relative variation $\Delta^{r} \chi$ of the secant magnetic susceptibility in the stress plane for a magnetic loading along RD (a) and at $45^{\circ}$ (b) - H=1000A/m.

Considering first an applied magnetic field along RD (Fig.1(a) and 2(a)), a uniaxial tension along the magnetic field direction improves the susceptibility and a compression in the same direction deteriorates the magnetic behaviour. When a uniaxial stress is applied in the direction perpendicular to the magnetic field, the effect is opposite but significantly softened compared to the parallel direction. A compression along the magnetic field direction always tends to deteriorate the magnetic behaviour whatever the value of $\sigma_{2}$. The isovalues for the susceptibility are almost vertical lines. The lowest values of $\chi$ are obtained for shear configurations with compression along the loading direction. A bi-tension tends to slightly increase the magnetic susceptibility especially at high field level. Variations are lower at high field since the material is closer to saturation. When the magnetic field is applied at $45^{\circ}$, isovalues seem to operate a rotation at $45^{\circ}$ too. This is particularly visible at low field. Indeed a symmetry with respect to the $\sigma_{1}=\sigma_{2}$ axis is expected since the material is transversely isotropic.

\section{Influence of a biaxial stress on the hysteretic behaviour}

The relative variation $\Delta^{r} H_{c}$ of the coercive field $H_{c}$ under biaxial loading is given in Fig.3 for the two magnetic field loading directions. These variations are calculated with respect to the unstressed configuration according to (2).

$$
\Delta^{r} H_{c}=100 \times \frac{H_{c}\left(\sigma_{1}, \sigma_{2}\right)-H_{c}(0,0)}{H_{c}(0,0)}=100 \times \frac{\Delta H_{c}}{H_{c}^{0}}
$$

For $\vec{H} / / R D$ (Fig.3a) $\Delta^{r} H_{c}$ is symmetric with respect to the shear line $\sigma_{1}=-\sigma_{2}$. The region of minimum coercive field is the region corresponding to shear stress with positive stress component along the rolling direction $\left(\sigma_{1}>0, \sigma_{2}<0\right)$. Along the line of pure shear stress $\left(\sigma_{1}=-\sigma_{2}\right)$ with $\sigma_{1}<0$, there is a strong increase of $H_{c}$ with the intensity of stress. The area of minimum effect of stress is the equibiaxial region close to the line $\sigma_{1}=\sigma_{2}$. The results obtained for a magnetic field applied along $R D$ are in very good agreement with the results obtained by Pearson et al. [3] at medium frequency $(50 \mathrm{~Hz})$ on $1 \mathrm{~mm}$ thick sheets of pure iron. In accordance with observations made on the magnetic susceptibility, the change of magnetic loading direction from $R D$ to $45^{\circ}$ leads to a rotation of

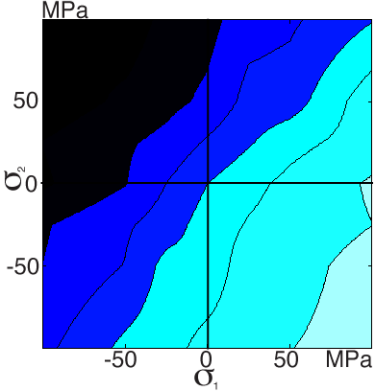

(a) $\vec{H} / / R D$

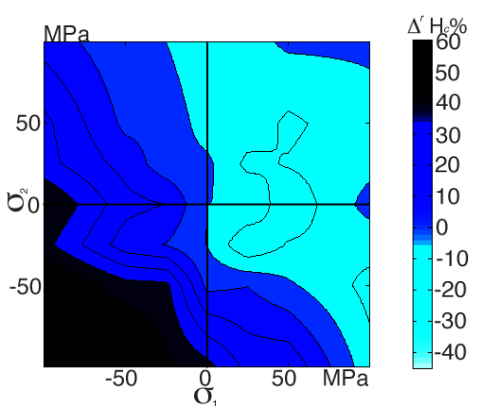

(b) $\vec{H} / / 45^{\circ}$
Fig. 3. Relative variation $\Delta^{r} H_{c}$ of the coercive field in the stress plane for a magnetic loading along $\mathrm{RD}$ (a) and at $45^{\circ}$ (b) - $f=5 \mathrm{~Hz}$.

the isovalues of coercive field. The figure becomes clearly symmetric with respect to the $\sigma_{1}=\sigma_{2}$ axis as expected for a transversely isotropic material under such loading conditions. The relative variation $\Delta^{r} P$ of the power losses $P$ under biaxial loading is reported in Fig.4 for the two magnetic field loading directions. These variations are calculated with respect to the unstressed configuration according to (3).

$$
\Delta^{r} P=100 \times \frac{P\left(\sigma_{1}, \sigma_{2}\right)-P(0,0)}{P(0,0)}=100 \times \frac{\Delta P}{P^{0}}
$$

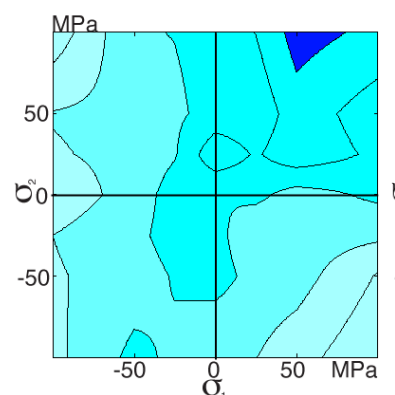

(a) $\vec{H} / / R D$

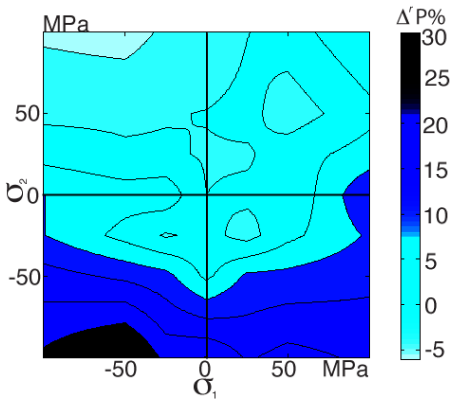

(b) $\vec{H} / / 45^{\circ}$
Fig. 4. Relative variation $\Delta^{r} P$ of the power losses in the stress plane for a magnetic loading along $\mathrm{RD}$ (a) and at $45^{\circ}$ (b) $-f=5 \mathrm{~Hz}$.

For $\vec{H} / / R D$ the effect of stress on the power losses is low compared to the effect on the coercive field or on the magnetic susceptibility at low field. A bi-tension state tends to increase the magnetic power losses (by approximately $10 \%$ for $\sigma_{1}=\sigma_{2}=100 \mathrm{MPa}$ ). The highest power losses are obtained along the equibiaxial line $\sigma_{1}=\sigma_{2}$. For $\vec{H} / / 45^{\circ}$, similarly to the trends observed for the magnetic susceptibility, the isovalues of power losses seem to operate a rotation at $45^{\circ}$. The map can be interpreted as a set of horizontal lines, and the highest power losses are obtained for high negative $\sigma_{2}$. The figure is consequently not symmetric with respect to the $\sigma_{1}=\sigma_{2}$ axis which is in contradiction with the material symmetries and with the susceptibility and coercive field results. Note that the measurement uncertainty is high for this quantity (about 10\%) and it could explain the unexpected trends observed.

An attempt of relationship between losses, susceptibility and coercive field evolutions under stress can be proposed. 
Assuming parallelogram-shaped cycles, the coercive field defines the width of magnetic cycles. On the other hand the height of the magnetic cycle will be proportional to the magnetic susceptibility (for a given maximum magnetic field). Losses variation is then the result of combined variations of coercive field and susceptibility. If power losses $P$ are assumed to be proportional to the product of coercive field with magnetisation, the relative variation of losses is linked to the sum of the relative variations of coercive field and susceptibility (4).

$$
P \propto H_{c} M=H_{c} \chi H, \quad \Delta^{r} P \propto \Delta^{r} H_{c}+\Delta^{r} \chi
$$

As a first approximation, experimental results seem to be in accordance with this assumption: at a given stress point $\left(\sigma_{1}, \sigma_{2}\right)$, a change (increase or decrease) of susceptibility seems to correspond to an opposite change (decrease or increase respectively) of coercive field. This leads to a relative insensitivity of losses to stress.

\section{EQUIVALENT STRESS FOR ANHYSTERETIC AND HYSTERETIC BEHAVIOUR}

Apart from a few noticeable exceptions (e.g. [4], [5], [6]), the coupled constitutive laws introduced in finite element modelling codes for rotating machines are limited to the restrictive case of uniaxial applied stress. The experimental results presented in the previous section show that this is usually irrelevant since the stress experienced by rotors is multiaxial. The use of equivalent stress approaches is a solution to overcome the limitations of existing uniaxial modelling tools [7]. In the case of magneto-elastic behaviour, the equivalent stress for a given multiaxial loading $\sigma$ is defined as the uniaxial stress $\sigma_{e q}$ - applied in the direction parallel to the magnetic field - that leads to the same magnetic behaviour than the actual multiaxial loading.

\section{A. Equivalent stress for anhysteretic behaviour}

A definition of an equivalent stress for anhysteretic magneto-elastic behaviour has been proposed recently [1]. It relies on an energy definition of the equilibrium. Based on an equivalence in magnetisation $\left(\vec{M}(\sigma)=\vec{M}\left(\sigma_{e q}\right)\right)$, the following definition of the equivalent stress $\sigma_{e q}$ is obtained:

$$
\sigma_{e q}=\frac{2}{3 k} \ln \left(\frac{2 \exp \left(\frac{3}{2} k \vec{h} \cdot \mathbf{s} \cdot \vec{h}\right)}{\exp \left(\frac{3}{2} k \vec{t}_{1} \cdot \mathbf{s} \cdot \vec{t}_{1}\right)+\exp \left(\frac{3}{2} k \vec{t}_{2} \cdot \mathbf{s} \cdot \vec{t}_{2}\right)}\right)
$$

$\mathbf{s}=\sigma-\frac{1}{3} \operatorname{tr}(\sigma) \mathbf{I}$ is the stress deviator, $\mathbf{I}$ is the identity tensor. The expression of $\sigma_{e q}$ includes a material parameter $k=3 \chi_{0} \lambda_{m} /\left(\mu_{0} M_{s}^{2}\right)$, with $\chi_{0}$ the initial susceptibility of the material, $\lambda_{m}$ the maximal magnetostriction (before magnetisation rotation within the magnetic domains), $\mu_{0}$ the vacuum permeability and $M_{s}$ the saturation magnetisation. $\left(\vec{h}, \vec{t}_{1}, \vec{t}_{2}\right)$ is a local coordinate system: $\vec{h}$ indicates the direction of the applied field, $\vec{t}_{1}$ is orthogonal to $\vec{h}$ and belongs to the sheet plane, and $\vec{t}_{2}$ is orthogonal to the sheet plane. In the configurations studied in this paper, the expression is given by (6) and (7) when the magnetic field is applied along $R D$ and at $45^{\circ}$ respectively.

$$
\begin{gathered}
\sigma_{e q}^{D L}=\frac{2}{3 k} \ln \left(\frac{2 \exp \left(\frac{3}{2} k \sigma_{1}\right)}{1+\exp \left(\frac{3}{2} k \sigma_{2}\right)}\right) \\
\sigma_{e q}^{45}=\frac{2}{3 k} \ln \left(\frac{2 \exp \left(\frac{3}{4} k\left(\sigma_{1}+\sigma_{2}\right)\right)}{1+\exp \left(\frac{3}{4} k\left(\sigma_{1}+\sigma_{2}\right)\right)}\right)
\end{gathered}
$$

Eq. (7) shows the expected symmetry with respect to the $\sigma_{1}=\sigma_{2}$ axis. Fig.5 illustrates the value of the equivalent stress $\sigma_{e q}$ according to this criterion with $k=18 \times 10^{-9} \mathrm{~m}^{3} / \mathrm{J}$ for this material.

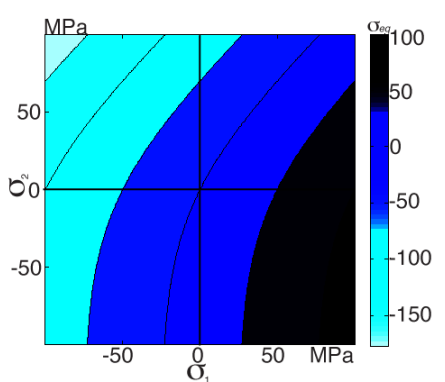

(a) $\vec{H} / / R D$

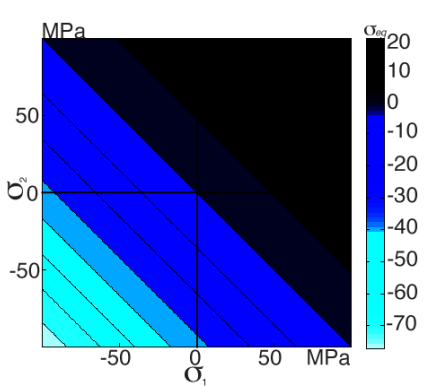

(b) $\vec{H} / / 45^{\circ}$
Fig. 5. Equivalent stress $\sigma_{e q}$ in the stress plane.

The behaviour of the material under uniaxial stress applied parallel to the magnetic field can be identified from Fig.1(a) (horizontal line $\sigma_{2}=0$ ). This is used as an input and, combined with the definition (5) of the equivalent stress, allows predicting the evolution of the magnetic susceptibility in the stress plane as plotted in Fig.6. The agreement between experiment (Fig.1) and modelling (Fig.6) is satisfactory. The same conclusions could be made at $1000 \mathrm{~A} / \mathrm{m}$ - using the uniaxial data from Fig.2(a). The equivalent stress criterion takes suitably into consideration the relative orientation of stress and magnetic field.

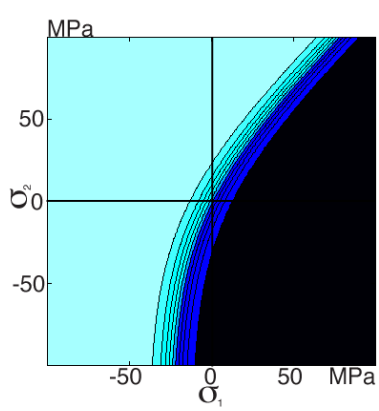

(a) $\vec{H} / / R D$

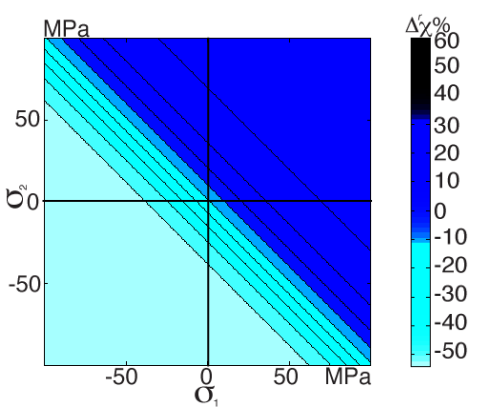

(b) $\vec{H} / / 45^{\circ}$
Fig. 6. Prediction of the relative variation of the secant magnetic susceptibility in the stress plane for a magnetic loading along $\mathrm{RD}$ (a) and at $45^{\circ}$ (b) - $\mathrm{H}=200 \mathrm{~A} / \mathrm{m}$.

\section{B. Equivalent stress for hysteretic behaviour}

The dissipation can be introduced by adding an irreversible contribution to the magnetic field, as proposed by Hauser 
under no applied stress [8]. Based on this definition, the dependence of the coercive field to the applied stress is defined in this paper thanks to the notion of "configuration effect". It comes from the simple observation of the initial domain structure modification when an external stress is applied: under tension, the fraction of domains oriented along the direction of the applied stress increases (positive magnetostriction) leading to a higher demagnetising effect and therefore to a refinement of the domain structure; under compression, the effect is opposite. The coercive field is associated to this configuration because the probability of encountering a pinning centre depends on the space between domain walls. The coercive field decreases with a tension aligned with the magnetic field; it increases under compression. We propose the following definition for the coercive field as a function of the multiaxial stress $\sigma$ :

$H_{c}=H_{c 0}\left(1-\eta\left(\frac{\exp (K \vec{h} \cdot \sigma \cdot \vec{h})}{\exp (K \vec{h} \cdot \sigma \cdot \vec{h})+\exp \left(K \vec{t}_{1} \cdot \sigma \cdot \vec{t}_{1}\right)+\exp \left(K \vec{t}_{2} \cdot \sigma \cdot \vec{t}_{2}\right)}-\frac{1}{3}\right)\right)$

$K$ and $\eta$ are material parameters. Using the identity $H_{c}(\sigma)=H_{c}\left(\sigma_{e q}^{c}\right)$, the equivalent stress for coercive field is given by (9).

$$
\sigma_{e q}^{c}=\frac{1}{K} \ln \left(\frac{2 \cdot \exp \left(K^{t} \vec{h} \mathbf{s} \vec{h}\right)}{\exp \left(K^{t} \vec{t}_{1} \mathbf{s} \vec{t}_{1}\right)+\exp \left(K^{t} \vec{t}_{2} \mathbf{s} \vec{t}_{2}\right)}\right)
$$

Due to the configuration effect expression, the equivalent stress for the coercive field is the same as the anhysteretic equivalent stress, except for the constant $K$ used as a fitting parameter. This formulation has been applied to describe the evolution of the coercive field under stress using the uniaxial results provided by the horizontal line $\sigma_{2}=0$ of Fig.3(a) as an input data (with $K=4 \times 10^{-9} \mathrm{~m}^{3} / \mathrm{J}$ for iron-silicon). The corresponding results are shown in Fig.7.

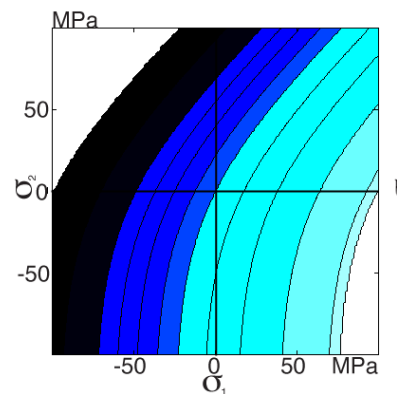

(a) $\vec{H} / / R D$

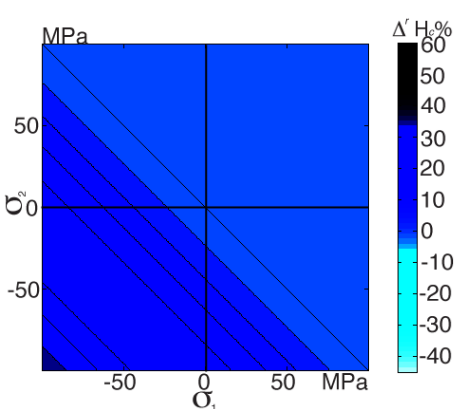

(b) $\vec{H} / / 45^{\circ}$
Fig. 7. Prediction of coercive field relative variation in the stress plane.

The results are satisfactory but reveal some shortcomings : when the applied magnetic field is along $R D$ (Fig.3(a) versus Fig.7(a)), the symmetry with respect to the $\sigma_{1}=-\sigma_{2}$ axis is not modelled; when the magnetic field is applied at $45^{\circ}$ (Fig.3(b) versus Fig.7(b)), the expected symmetry is observed but the coercive field decrease in bi-tension is hardly observed.

The effect of stress on power losses can be defined as a combination of the effect on magnetic susceptibility and on coercive field using (4). Fig.8 gives the corresponding variations of power losses in the stress plane predicted from this approach. The horizontal lines $\sigma_{2}=0$ of Fig.1(a) and 3(a) are used as an input to build this graph. As experimentally observed, a weak level of variation is modelled especially for $\vec{H} / / R D$. The highest loss levels are obtained in bi-compression for $\vec{H} / / 45^{\circ}$. The trends observed for $\vec{H} / / R D$ between experiments and model are nevertheless different. This could be due to the high uncertainty level for power losses measurements and to the strong assumptions in the modelling.

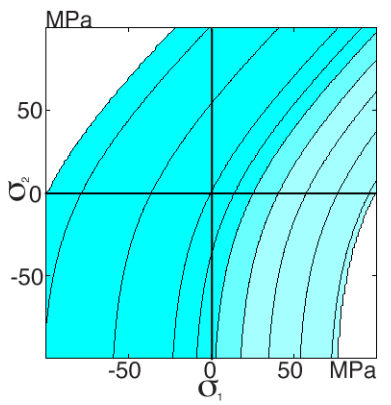

(a) $\vec{H} / / R D$

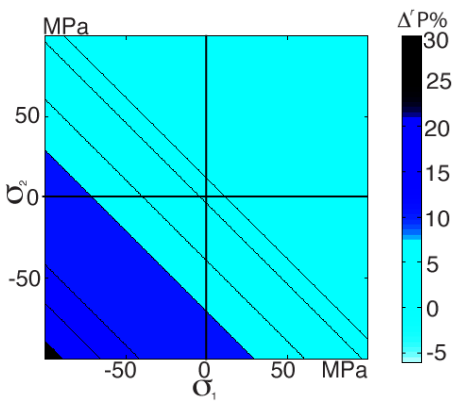

(b) $\vec{H} / / 45^{\circ}$
Fig. 8. Prediction of power losses relative variation in the stress plane.

\section{CONCLUSION}

An equivalent stress for hysteresis losses is proposed in this paper. It is a combination of an anhysteretic and a coercive field equivalent stress, both based on a very similar formulation. Some limitations have been highlighted, notably regarding the $\sigma_{1}=-\sigma_{2}$ symmetry axis for $\vec{H} / / R D$. The assumption of parallelogram-shaped cycles is also very strong. This approach is nevertheless a first step towards the introduction of the concept of equivalent stress for the prediction of iron losses under magneto-mechanical loading.

\section{REFERENCES}

[1] O. Hubert, L. Daniel, Energetical and multiscale approaches for the definition of an equivalent stress for magneto-elastic couplings, J Magn. Magn. Mater. 323(13)(2011), 1766-1781.

[2] M. Rekik, O. Hubert, L. Daniel, Influence of a multiaxial stress on the reversible and irreversible magnetic behaviour of a $3 \% \mathrm{Si}-\mathrm{Fe}$ alloy, Int. J. Appl. Electr. Mech. (2013) accepted for publication.

[3] J. Pearson, P.T. Squire, M.G. Maylin, J.G. Gore, Biaxial Stress Effects on the Magnetic Properties of Pure Iron, IEEE Trans. Magn. 36(2000), 3251-3253.

[4] K. Fonteyn, A. Belahcen, R. Kouhia, P. Rasilo, A. Arkkio, FEM for Directly Coupled Magneto-Mechanical Phenomena in Electrical Machines, IEEE Trans. Magn. 46(8)(2010), 2923-2926.

[5] L. Bernard, X. Mininger, L. Daniel, G. Krebs, F. Bouillault, M. Gabsi, Effect of stress on switched reluctance motors: a magneto-elastic finiteelement approach based on multiscale constitutive laws, IEEE Trans. Magn. 47(9)(2011), 2171-2178.

[6] S. Zeze, Y. Kai, T. Todaka, M. Enokizono, Vector Magnetic Characteristic Analysis of a PM Motor Considering Residual Stress Distribution With Complex-Approximated Material Modeling, IEEE Trans. Magn. 48(11)(2012), 3352-3355.

[7] G. Krebs, L. Daniel, Giant magnetostrictive materials for field weakening: a modeling approach, IEEE Trans. Magn., 48(9)(2012), 2488-2494.

[8] H. Hauser, Energetic model of ferromagnetic hysteresis: Isotropic magnetization, J. Appl. Phys. , 96 (5) (2004), 2753-2767. 